\title{
Optimization of the Initial Parameters of the Utilization Circuit of Cogeneration Combined Cycle Plant of Two Pressure Levels
}

\author{
Kalyutik A.A., Treshchev D.A., Treshcheva M.A. \\ Peter the Great St. Petersburg Polytechnic University \\ Saint Petersburg, Russian Federation
}

\begin{abstract}
This article is devoted to optimization of parameters and structure of the industrial-heating vapor-gas facilities (HVGF). The aim of the study is determination of the optimal initial parameters of two-circuit utilization HVGF. The set goal is achieved based on the methods of the mathematical modeling of the thermal schemes, using the elements of thermodynamic economic analysis. As the optimization criterion, the index of a relative fuel economy is used upon the combined generation of the energy and heat. The most significant result of the research consists in revealing a tendency to an increase in the optimal vapor pressure of the HVGF high pressure circuit, compared to a similar parameter of the condensation vapor gas device (VGD CEPP). For the two-circuit HVGF, the optimal initial pressure is 9.8-10 MPa (for the CEPP it is $8 \mathrm{MPa}$ ). The tendency is revealed for an increase of the relative fuel economy upon the complication of the HVGF thermal scheme. It was established that for the identic HVGF located in different energy systems, the relative fuel economy differ greatly. The relative fuel economy for the HVGF under consideration compared to analogous VGD CEPP is 27.35 $\%$, and compared to the vapor turbine CEPP, the relative fuel economy will increase by $8.8 \%$. The importance of the results obtained consists in that their application will make it possible to increase the energy efficiency of the HVGF being constructed, and optimize the structure of the energy systems of separate regions and states.
\end{abstract}

Keywords: thermoelectric power plant, heating, cogeneration plant, combined cycle power plant, fuel economy.

DOI: https://doi.org/10.52254/1857-0070.2021.1-49.06

UDC: 621.438

Optimizarea parametrilor inițiali ai circuitului de utilizare a unei unități de cogenerare CCGT cu două niveluri de presiune

Kaliutik A.A., Trescev D.A., Tresceva M.A.

Petru cel Mare Universitatea Politehnică din Sankt Petersburg

Saint-Petersburg, Federația Rusă

Rezumat. Articolul este dedicat optimizării parametrilor și structurii plante cu ciclu combinat de cogenerare (PCCC). Scopul studiului este de a determina parametrii iniţiali optimi ai unui tip de utilizare PCCC cu circuit dublu cu unități de turbină cu gaz (TCG) din clasa E. Acest obiectiv este atins pe baza metodelor de modelare matematică a circuitelor termice ale PCCC, utilizând elemente de analiză termodinamică și economică. Ca criteriu de optimizare, se utilizează indicatorul economiei relative de combustibil în generarea combinată de energie și căldură. Utilizarea acestui indicator se datorează corespondenței valorii sale maxime cu creșterea maximă a efectului economic integral din generația combinată. Cel mai semnificativ rezultat al cercetării este identificarea unei tendințe către creșterea presiunii optime de vapori a circuitului de înaltă PCCC cu două circuite, presiunea iniţială optimă, determinată utilizând indicatorul economiei relative de combustibil, este de 9,8-10 MPa (pentru stații electrice de condensare(SEC)), presiunea inițială optimă a aburului este de $8 \mathrm{MPa}$ ). Se dezvăluie o tendință de creștere a economiei relative de combustibil, odată cu complicația circuitului termic al PCCC. Economia relativă de combustibil pentru PCCC considerată în comparație cu TCG SEC similar este de 27,35\%, iar în comparație cu turbina cu abur SEC, pe baza unității de serie K-300-240, va crește cu 8,8\%. Semnificația rezultatelor obținute constă în faptul că utilizarea lor, în etapa de proiectare, va crește eficiența energetică a PCCC-urilor în construcție, precum și va optimiza structura sistemelor energetice din regiuni și state individuale.

Cuvinte-cheie: CET, termoficare, instalație de cogenerare, instalație de gaze și abur, economisirea combustibilului.

СКалютик А.А., Трещёв Д.А., Трещёва М.А. 2021. 


\section{Оптимизация начальных параметров утилизационного контура теплофикационной ПГУ двух уровней давления}

Калютик А.А., Трещёв Д.А., Трещёва М.А.

Санкт-Петербургский политехнический университет Петра Великого

Санкт-Петербург, Российская Федерация

Аннотация. Статья посвящена проблематике оптимизации параметров и структуры теплофикационных парогазовых установок (ТПГУ). Целью исследования является определение оптимальных начальных параметров двухконтурных ТПГУ утилизационного типа с газотурбинными установками (ГТУ) Е-класса. Поставленная цель достигается на базе методов математического моделирования тепловых схем ТПГУ, с использованием элементов термодинамического и экономического анализа. В качестве критерия оптимизации используется показатель относительной экономии топлива при комбинированной выработке электроэнергии и тепла. Применение данного показателя обусловлено соответствием его максимального значения максимуму приращения интегрального экономического эффекта от комбинированной выработки. Наиболее существенный результат исследований заключается в выявлении тенденции к увеличению оптимального давления пара контура высокого давления ТПГУ, по сравнению с аналогичным параметром конденсационной парогазовой установки (ПГУ КЭС). Для двухконтурной ТПГУ с ГТУ Е-класса, оптимальное начальное давление, определенное с помощью показателя относительной экономии топлива, составляет 9.8-10 МПа (для КЭС оптимальное начальное давление пара $8 \mathrm{MПа).} \mathrm{Выявлена} \mathrm{тенденция} \mathrm{к} \mathrm{увеличению} \mathrm{относительной} \mathrm{экономии} \mathrm{топлива} \mathrm{при} \mathrm{усложнении}$ тепловой схемы ТПГУ. Для одноконтурной ТПГУ относительная экономия составляет $26.43 \%$, а для двухконтурной ТПГУ - 27.35\%. Установлено, что для аналогичных двухконтурных ТПГУ, расположенных в различных энергосистемах, величина относительной экономии топлива и приращение интегрального экономического эффекта, существенно отличается. Относительная экономия топлива для рассматриваемой ТПГУ по сравнению с аналогичной ПГУ КЭС составляет $27.35 \%$, а по сравнению с паротурбинной КЭС, на базе серийного блока К-300-240, увеличится на 8.8\%. Значимость полученных результатов состоит в том, что их использование, на стадии проектирования, позволит повысить энергетическую эффективность строящихся ТПГУ, а также оптимизировать структуру энергосистем отдельных регионов и государств.

Ключевые слова: теплоэлектростанция, теплофикация, когенерационная установка, парогазовая установка, экономия топлива.

\section{ВВЕДЕНИЕ}

В настоящее время, динамичное социально-экономическое развитие государства невозможно без решения серьезной комплексной задачи, связанной с переходом всех ключевых отраслей экономики на качественно новый уровень [1-3]. Анализ ситуации, на мировых рынках энергоресурсов показывает, что топливноэнергетические комплексы (ТЭК) как развивающихся, так и развитых стран, в перспективе будет сталкиваться со сложным комплексом внутренних и внешних вызовов, оказывающим значительное воздействие на развитие и рост крупных энергетических компаний [4$6]$.

В качестве одного из основных критериев эффективности деятельности энергетической компании выступает ее стоимость. На рыночной оценке российских энергетических компаний негативно сказывается, сложившаяся к настоящему моменту, структура генерирующих мощностей, характеризующаяся значительной долей морально и технически устаревшего

оборудования, нуждающегося в замене или модернизации $[3,6,7]$.

В качестве основных факторов, способствующих увеличению стоимости энергокомпаний, в том числе, выступает повышение эффективности реализации проектов технического перевооружения и реконструкции, строительство новых мощностей на базе наиболее современных экономичных технологий, повышение эффективности действующего бизнеса и инвестиционной деятельности [7-9]. Например, к основным мероприятиям, направленным на повышение энергетической эффективности, за счет которых в период 2015-2018 гг. была достигнута значительная экономия топливно-энергетических ресурсов крупными российскими энергокомпаниями (такими как ПАО «Мосэнерго», ПАО «Т Плюс», АО «ТГК-16», ПАО «Интер РАО», ПАО «ОГК-2»), относится: оптимизация производства электроэнергии на более энергоэффективном оборудовании, а также вывод устаревшего оборудования и ввод 
нового на базе парогазовых технологий [10-

В настоящее время, тепловые электростанции на базе парогазовых установок утилизационного типа являются наиболее экономичным, с точки зрения производства электроэнергии, а также обладают высокими экологическими показателями [9]. Именно поэтому значительная доля генерирующего оборудования, вводимого в эксплуатацию в России, основана на использовании ПГУ [2, $5,11,12]$. Как в России, так и за рубежом ведется активная научная работа по анализу эффективности действующих, вновь вводимых в эксплуатацию и проектируемых ПГУ [13-22]. Разрабатываются новые схемные и технологические решения $[19,21,22,27]$, а также ведутся исследования в области оптимизации параметров и структуры ПГУ [23-29].

Существенным вопросом, возникающим на этапе проектирования когенерационных установок, является учет влияния комбинированной выработки на выбор и обоснование оптимальных начальных параметров.

Системным исследованиям в области оптимизации паросиловых ТЭЦ, с использованием в качестве критерия показателя относительной экономии топлива по сравнению с раздельным производством электроэнергии и тепла, были посвящены научные труды целого ряда крупных советских и российских ученых (Мелентьева Л.А., Хрилева Л.С., Андрющенко А.И., Соколова Е.Я. и др.) [30]. Однако для ТЭЦ с ПГУ утилизационного типа оптимизация параметров и структуры осуществлялось преимущественно на базе решения частных задач, связанных с исследованием влияния отдельных термодинамических параметров на показатели тепловой эффективности конкретного схемного решения без учета влияния когенерации $[29,30,34]$.

В статье [30] была обоснована возможность применения критерия относительной экономии топлива по сравнению с раздельным производством электрической и тепловой энергией для оптимизации начальных параметров ТПГУ в современных экономических условиях, на
12]. примере одноконтурной ТПГУ на базе GT13E2.

Также было показано, что в теплофикационной ПГУ утилизационного типа с одним контуром давления оптимальное начальное давление, рассчитанное с использованием относительной экономии топлива, смешается в сторону увеличению относительно оптимального значения для ПГУ-КЭС на базе аналогичной ГТУ.

Однако, оптимизация ТПГУ утилизационного типа более сложной структуры, с применением относительной экономии топлива, ранее не проводились.

Цель настоящего исследования оценить оптимальные начальные параметры в утилизационном контуре ТПГУ двух уровней давления пара с использованием показателя относительной экономии топлива по сравнению с раздельной выработкой электрической и тепловой энергий.

\section{МЕТОДЫ ИССЛЕДОВАНИЯ}

При выборе параметров и структуры когенерационных установок в качестве основного критерия может выступать экономия топлива при комбинированной выработке, по сравнению с раздельной [30]. Это обусловлено тем, что изменение начальных параметров пара утилизационного контура ТПГУ не приводит к существенному изменению капитальных вложений, до $80 \%$ эксплуатационных издержек приходится на топливные затраты [31-32], а постоянная составляющая эксплуатационных издержек в первом приближении может быть задана в долях от капитальных вложений в энергоустановку данного типа [31-32].

В этом случае, при выполнении условия равенства объемов производства электрической и тепловой энергий в комбинированной и раздельной схеме, справедливо следующее выражение (1):

$$
\left\{\begin{array}{l}
\overline{\Delta Q}_{\text {mon }} \rightarrow \text { мax } \\
\sum_{t=0}^{T} \Delta H_{t} /\left(1+E_{н}\right)^{t} \rightarrow \max (1) \\
\text { АДД } \rightarrow \text { мax }
\end{array}\right.
$$


где $\overline{\Delta Q}_{\text {mon }}-$ относительная экономия топлива в ТПГУ по сравнению с раздельной выработкой; $\Delta h_{t}$ - экономия ежегодных издержек за счет увеличения эффективности по сравнению с раздельной выработкой; $T$ - срок службы энергоустановки; $E_{н}$ - норма дисконта; АЧДД - чистый дисконтированный доход или интегральный экономический эффект от комбинированной выработки за весь период службы ТПГУ.

Формула для определения относительной экономии топлива по сравнению с раздельной выработкой для ТПГУ, имеет следующий вид [30] (2):

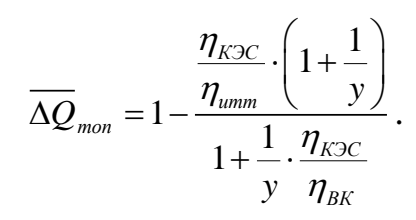

где $\eta_{B K}-$ КПД замещающей водогрейной котельной; $\eta_{\text {кэс }}$ - КПД замещающей КЭС; $\eta_{\text {umm }}$ - коэффициент использования теплоты топлива; $y-$ удельная выработка электроэнергии на тепловом потреблении.

С помощью показателя относительной экономии топлива по сравнению с раздельной выработкой была проведена оптимизация начального давления пара для двухконтурной ТПГУ с ГТУ Е-класса (принципиальная схема представлена на рисунке 1). В качестве примера ГТУ Екласса в статье рассматривается установка GT13E2 (основные характеристики приведены в таблице 1).

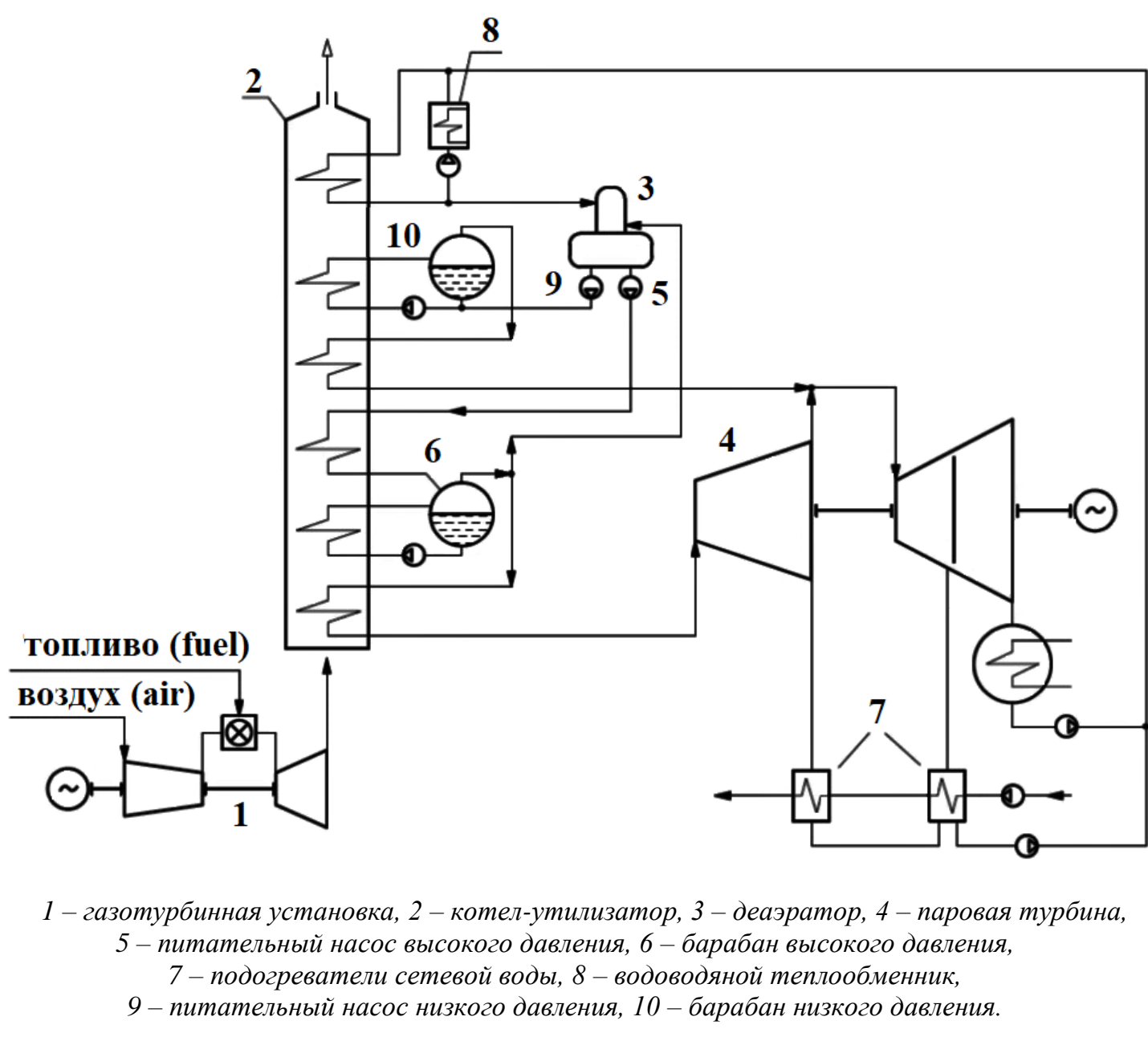

Рис. 1. Принципиальная схема двухконтурной теплофикационной парогазовой установки ${ }^{1}$

\footnotetext{
${ }^{1}$ Appendix 1
} 
Таблица 1.

Показатели газовой турбины GT13E2 при температуре наружного воздуха $-1,3^{\circ} \mathrm{C}^{2}$

\begin{tabular}{|c|c|}
\hline $\begin{array}{c}\text { Показатель } \\
\text { (Indicator) }\end{array}$ & $\begin{array}{c}\text { Значение } \\
\text { (Value) }\end{array}$ \\
\hline $\begin{array}{c}\text { Электрическая мощность } \\
\text { (Electric power), MW }\end{array}$ & 196.1 \\
\hline $\begin{array}{c}\text { КПД } \\
\text { (Efficiency), \% }\end{array}$ & 37.3 \\
\hline $\begin{array}{c}\text { Температура } \\
\text { уходящих газов } \\
\text { (Exhaust gas temperature), } \\
{ }^{\circ} \mathrm{C}\end{array}$ & 504 \\
\hline $\begin{array}{c}\text { Рacход уходящих газов } \\
\text { (Exhaust gas flow), } \\
\mathrm{kg} / \mathrm{s}\end{array}$ & 594 \\
\hline
\end{tabular}

При проведении математического моделирования тепловой схемы двухконтурной ТПГУ утилизационного типа были приняты следующие исходные данные и условия:

- расчетная температура номинального режима принята равной средней температуре отопительного периода в г. Санкт-Петербурге (на уровне $-1.3^{\circ} \mathrm{C}$ ) [33];

- диапазон изменения параметров второго контура котла-утилизатора ограничен и, при проведении расчетов, давление и температура пара в контуре низкого давления приняты на уровне 0.7 МПа и $200{ }^{\circ} \mathrm{C}$, соответственно;

- в рассматриваемой тепловой схеме утилизационного контура, установлен деаэратор с рабочим давлением 0.6 МПа, а следовательно, экономайзер низкого давления котла-утилизатора вырожден и в расчетах не учтен;

- величины минимального температурного напора $\left(\Delta t_{\min }\right)$ и недогрева воды до температуры насыщения в экономайзере высокого давления котла-утилизатора приняты равными $10{ }^{\circ} \mathrm{C}$;

- начальная температура пара контура высокого давления принята равной $480{ }^{\circ} \mathrm{C}$, а начальное давление меняется в диапазоне, ограниченном уровнем допустимой влажности в конце процесса расширения пара в турбине (в пределах от 5 до $11 \mathrm{MПа);}$

\footnotetext{
${ }^{2}$ Appendix 1
}

- относительный внутренний КПД паровой турбины без учета потерь от влажности принят равным 0.9;

- давления в камерах нижнего и верхнего теплофикационных отборов приняты на $\begin{array}{llll}\text { уровне } & 0.026 \mathrm{M} \text { Па } & \text { и } & 0.089 \mathrm{M} \text { Па }\end{array}$ соответственно;

- температурный график теплосети $150 / 70^{\circ} \mathrm{C}$.

\section{РЕЗУЛЬТАТЫ И ОБСУЖДЕНИЕ}

Сводные результаты расчета основных режимных характеристик утилизационного контура и технико-экономических показателей двухконтурной теплофикационной ПГУ-260 (на базе ГТУ Е-класса, на примере GT13E2), при варьировании давления свежего пара контура высокого давления в пределах от 5 до 11 МПа, представлены в таблице 2 и таблице 3 соответственно.

Результаты проведенного исследования влияния начального давления на техникоэкономическую эффективность ТПГУ утилизационного типа, в графическом виде отражены на рисунках 2 и 3.

На рисунке 2 представлена зависимость коэффициента использования теплоты топлива и удельной выработки электроэнергии на тепловом потреблении от давления пара контура ВД для двухконтурной ТПГУ.

На рисунке 3 представлена зависимость относительной экономии топлива по сравнению с раздельной выработкой от начального давления пара контура ВД для одноконтурной и двухконтурной ТПГУ при $\eta_{\text {КэС }}=50.6 \%$ и $\eta_{B K}=94 \%$.

Варьирование давления пара контура ВД практически не оказывает влияния на коэффициент использования теплоты топлива двухконтурной ТПГУ (рис.2). Удельная выработка на тепловом потреблении и относительная экономия топлива по сравнению с раздельной выработкой достигают максимального значения при давлении пара контура ВД на уровне 9-10 МПа (рис.2).

При оптимизации двухконтурных конденсационных ПГУ утилизационного типа (на базе современных ГТУ), максимальный КПД цикла по выработке электроэнергии достигается, как показывают ранее проведенные исследования, при давлении пара контура ВД около 8 МПа [29, 34]. 
Таблица 2.

Сводные результаты расчета параметров режима двухконтурной теплофикационной парогазовой установки $^{3}$

\begin{tabular}{|c|c|c|c|c|c|c|c|}
\hline $\begin{array}{l}\text { Показатель } \\
\text { (Indicator) }\end{array}$ & \multicolumn{7}{|c|}{$\begin{array}{l}\text { Значение } \\
\text { (Value) }\end{array}$} \\
\hline \multicolumn{8}{|c|}{ Контур высокого давления (The high-pressure circuit) } \\
\hline $\begin{array}{c}\text { Давление пара } \\
\text { (Steam pressure), MPa }\end{array}$ & 5 & 6 & 7 & 8 & 9 & 10 & 11 \\
\hline $\begin{array}{c}\text { Температура пара } \\
\text { (Steam temperature), }{ }^{\circ} \mathrm{C}\end{array}$ & \multicolumn{7}{|c|}{480} \\
\hline $\begin{array}{c}\text { Относительный расход пара } \\
\text { (The relative consumption } \\
\text { of steam), } \\
\mathrm{kg} \text { of steam } / \mathrm{kg} \text { of gas }\end{array}$ & 0.1065 & 0.1042 & 0.1022 & 0.1004 & 0.0989 & 0.0975 & 0.0963 \\
\hline \multicolumn{8}{|c|}{ Контур низкого давления (The low-pressure circuit) } \\
\hline $\begin{array}{c}\text { Давление пара } \\
\text { (Steam pressure), MPa }\end{array}$ & \multicolumn{7}{|c|}{0.7} \\
\hline $\begin{array}{l}\text { Температура пара } \\
\text { (Steam temperature), }{ }^{\circ} \mathrm{C}\end{array}$ & \multicolumn{7}{|c|}{200} \\
\hline $\begin{array}{c}\text { Относительный расход пара } \\
\text { (The relative consumption } \\
\text { of steam), } \\
\mathrm{kg} \text { of steam } / \mathrm{kg} \text { of gas }\end{array}$ & 0.0269 & 0.0305 & 0.0336 & 0.0365 & 0.0391 & 0.0415 & 0.0437 \\
\hline \multicolumn{8}{|c|}{ Электрическая мощность (Electric power), MW } \\
\hline $\begin{array}{l}\text { Паровая турбина } \\
\text { (Steam Turbine), MW }\end{array}$ & 60.52 & 61.44 & 62.31 & 62.87 & 63.54 & 63.78 & 62.91 \\
\hline $\begin{array}{l}\text { Газовая турбина } \\
\text { (Gas Turbine), MW }\end{array}$ & 196.09 & 196.09 & 196.09 & 196.09 & 196.09 & 196.09 & 196.09 \\
\hline $\begin{array}{c}\text { Парогазовая установка } \\
\text { (Combined cycle gas plant), } \\
\text { MW }\end{array}$ & 256.61 & 257.53 & 258.41 & 258.97 & 259.64 & 259.88 & 259 \\
\hline \multicolumn{8}{|c|}{$\begin{array}{l}\text { Тепловая мощность в расчете на килограмм газа } \\
\text { (Heat output per kilogram of gas), KJ/kg of gas }\end{array}$} \\
\hline $\begin{array}{c}\text { Паровая турбина } \\
\text { (Steam Turbine), KJ/kg of gas }\end{array}$ & 284.75 & 283.88 & 283.06 & 282.75 & 282.25 & 282.44 & 284.43 \\
\hline $\begin{array}{c}\text { Водо-водяной } \\
\text { теплообменник } \\
\text { (Water-water heat exchanger), } \\
\text { KJ/kg of gas }\end{array}$ & 50.04 & 49.51 & 49.03 & 48.58 & 48.15 & 47.75 & 47.36 \\
\hline $\begin{array}{c}\text { Парогазовая установка } \\
\text { (Combined cycle gas plant), } \\
\text { KJ/kg of gas }\end{array}$ & 334.8 & 333.39 & 332.09 & 331.33 & 330.4 & 330.19 & 331.79 \\
\hline
\end{tabular}

\footnotetext{
${ }^{3}$ Appendix 1
} 
Расчеты показали, что максимальное значение относительной экономии топлива по сравнению с раздельной выработкой, в ТПГУ более сложной структуры (двухконтурной) достигается при более высоких начальных параметрах пара утилизационного контура, чем для аналогичной одноконтурной установки (рис. 3).

Для рассматриваемого класса установок, наибольшее значение относительной экономии от комбинированной выработки (по сравнению с аналогичной ПГУ-КЭС с эффективностью 50.6\%) для одноконтурной ТПГУ составляет 26.43 \% и достигается при начальном давление пара контура высокого давления на уровне 5.5-6 МПа.

Для двухконтурной ТПГУ (на базе ГТУ того же типа) показатель относительной экономии топлива составляет 27.35\% при начальном давлении пара контура высокого давления в 9.8-10 МПа.

Таблица 3.

Сводные результаты расчета показателей эффективности двухконтурной теплофикационной парогазовой установки

\begin{tabular}{|c|c|c|c|c|c|c|c|}
\hline $\begin{array}{c}\text { Показатель } \\
\text { (Indicator) }\end{array}$ & \multicolumn{7}{|c|}{$\begin{array}{c}\text { Значение } \\
\text { (Value) }\end{array}$} \\
\hline $\begin{array}{c}\text { Давление пара } \\
\text { контура высокого } \\
\text { давления } \\
\text { (Pressure of steam from } \\
\text { the high-pressure } \\
\text { circuit), MPa }\end{array}$ & 5 & 6 & 7 & 8 & 9 & 10 & 11 \\
\hline $\begin{array}{c}\text { Коэффициент } \\
\text { использования } \\
\text { теплоты топлива } \\
\text { (Coefficient of } \\
\text { utilization of heat of } \\
\text { fuel), \% }\end{array}$ & 86.74 & 86.76 & 86.78 & 86.8 & 86.82 & 86.85 & 86.86 \\
\hline $\begin{array}{c}\text { КПД цикла, } \\
\text { по выработке } \\
\text { электроэнергии } \\
\text { (Cycle efficiency), }\end{array}$ & 48.87 & 49.05 & 49.22 & 49.32 & 49.45 & 49.5 & 49.33 \\
\hline $\begin{array}{c}\text { Коэффициент } \\
\text { выработки } \\
\text { электроэнергии на } \\
\text { тепловом потреблении } \\
\text { (Coefficient of specific } \\
\text { electricity generation } \\
\text { based on heat } \\
\text { consumption) }\end{array}$ & 1.2906 & 1.3007 & 1.3102 & 1.3161 & 1.3232 & 1.3252 & 1.3144 \\
\hline $\begin{array}{c}\text { Относительная } \\
\text { экономия топлива при } \\
\text { комбинированном } \\
\text { производстве } \\
\text { электроэнергии и } \\
\text { тепла } \\
\text { (Relative fuel } \\
\text { economy), \% }\end{array}$ & 27.09 & 27.17 & 27.25 & 27.29 & 27.35 & 27.36 & 27.28 \\
\hline
\end{tabular}

\footnotetext{
${ }^{4}$ Appendix 1
} 


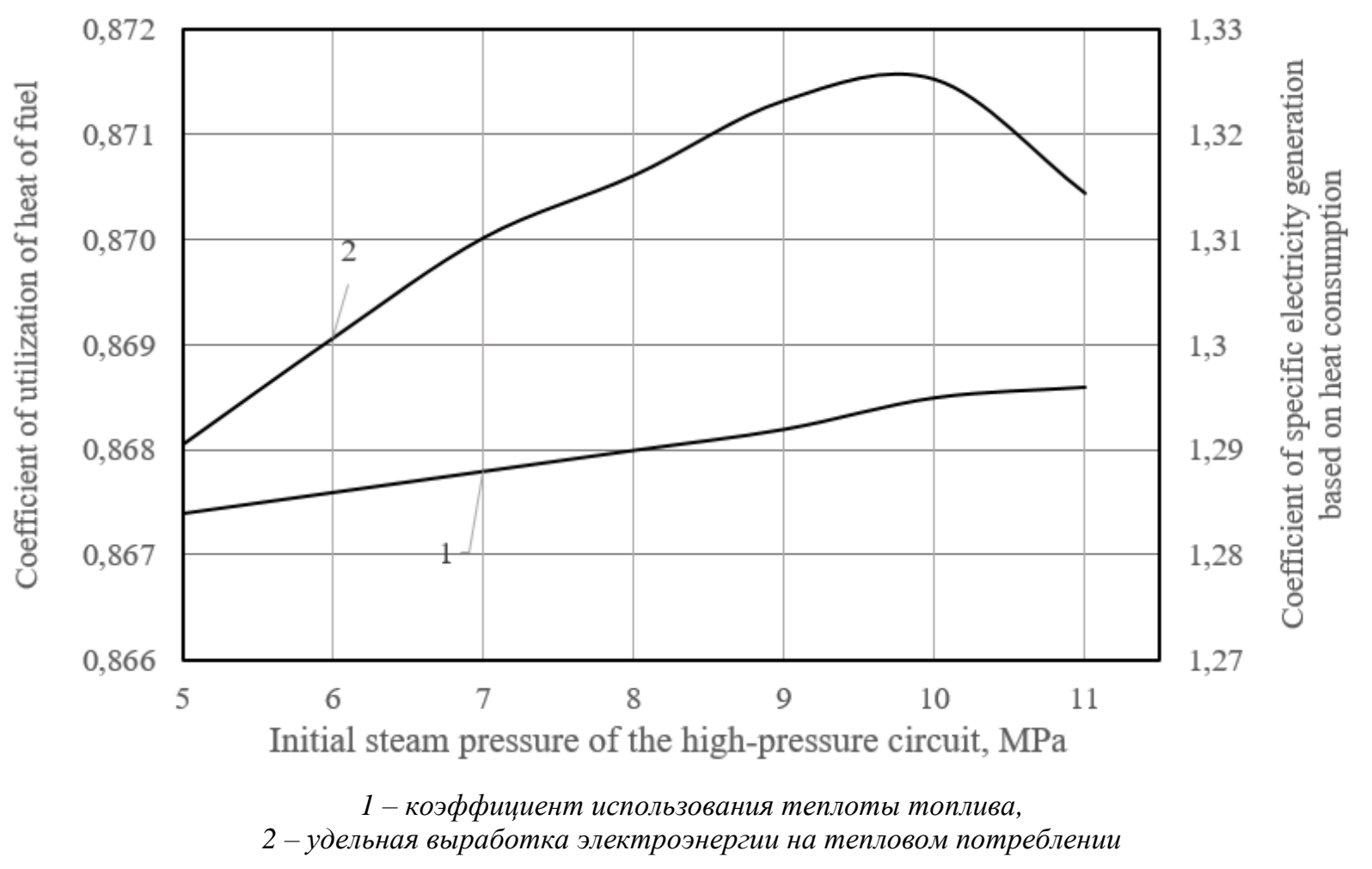

Рис.2. Показатели работы теплофикационной двухконтурной парогазовой установки ${ }^{5}$

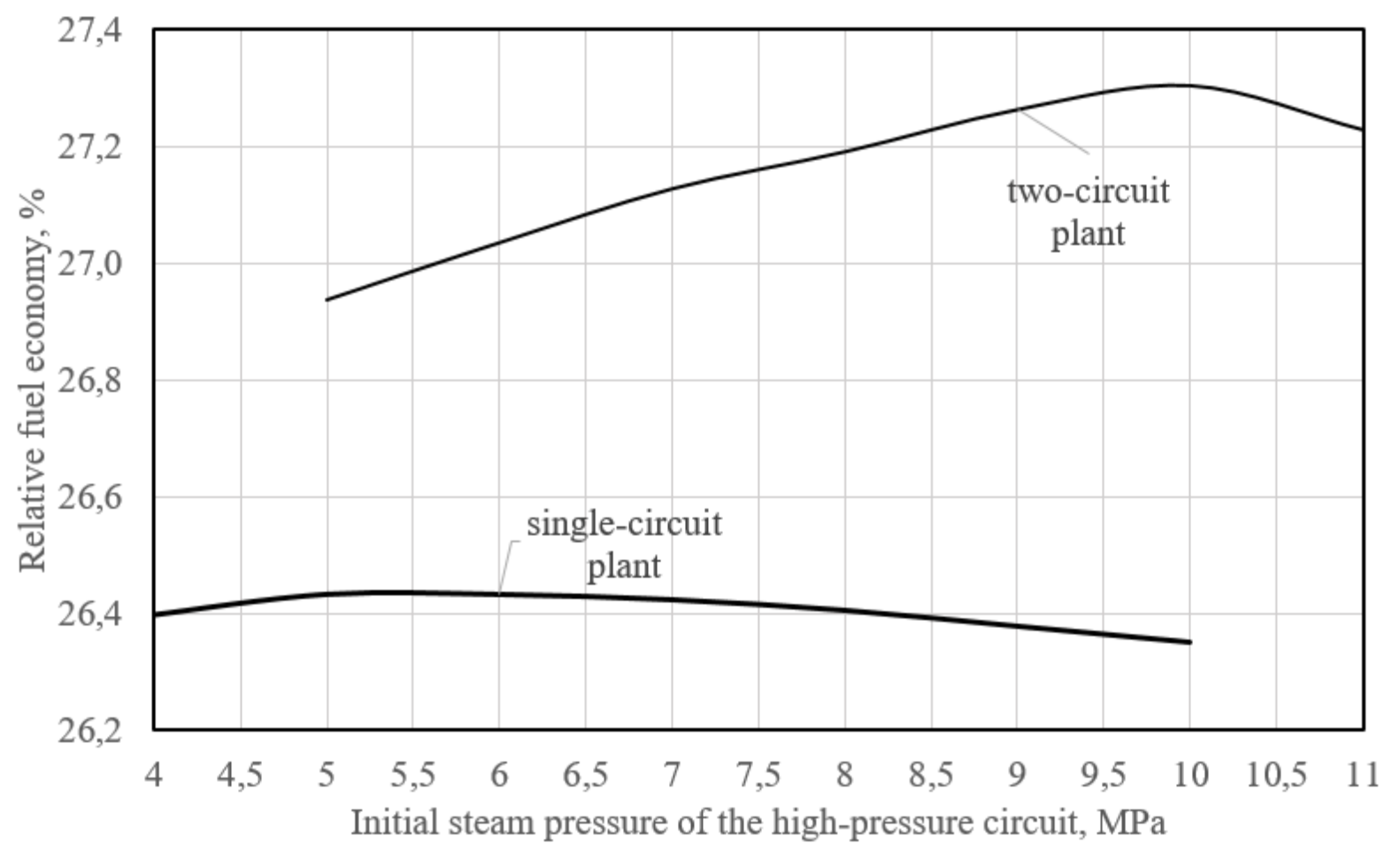

Рис.3. Относительная экономия топлива для одноконтурной и двухконтурной парогазовой установки 
Таким образом, с точки зрения максимизации экономического эффекта за счет минимизации топливной составляющей эксплуатационных затрат, усложнение структуры тепловой схемы ТПГУ должно сопровождаться увеличением начальных параметров пара.

Однако, в ряде случаев, может быть целесообразно применение одинаковых начальных параметров пара контура высокого давления, как для конденсационных, так и теплофикационных установок, с точки зрения унификации конструктивных решений по основному оборудованию утилизационного контура.

Зона оптимальных параметров пара ТПГУ достаточно пологая. Для двухконтурной теплофикационной ПГУ-260 (на базе GT13E2) с начальными параметрами 5-6 МПа снижение относительной экономии топлива составит до 0.4-0.5\%, по сравнению с аналогичной ТПГУ с начальным давление контура высокого давления 9-10 МПа.

Существенное влияние на величину относительной экономии топлива по сравнению с раздельной выработкой ТПГУ, оказывает эффективность замещающей КЭС (по сути, отражающей системные условия в которых функционирует электростанция). C уменьшением КПД замещающей КЭС, относительная экономия топлива в теплофикационной установке растет.

Так в условиях энергосистемы, основу которой составляют ТЭС с эффективностью на уровне $40 \%$ (например, на базе типовой установки с паровой турбиной К-300-240), при том же уровне эффективности замещающей водогрейной котельной, для одноконтурной ТПГУ утилизационного типа относительная экономия топлива составит $37.9 \%$, а для аналогичной двухконтурной ТПГУ - 38.8\%.

\section{ВЫВОДЫ}

1. При сравнении комбинированной и раздельной схем производства электрической и тепловой энергии на ПГУ ТЭС, если выполняется условие равенства объемов производства, а изменение параметров или структуры тепловой схемы не приводит к существенному изменению капитальных вложений в строительство энергоустановки, относительной экономии топлива от комбинированной выработки позволит максимизировать также чистый дисконтированный доход от двухконтурной ТПГУ.

2. Максимальное значение относительной экономии топлива по сравнению с раздельной выработкой в двухконтурной ТПГУ (на базе GT13E2) достигается при начальном давлении пара контура ВД на уровне 9.8-10 МПа (для аналогичной одноконтурной ТПГУ оптимальное начальное давление пара составляет 5.5-6 МПа).

3. Усложнение структуры ТПГУ ведет к увеличению относительной экономии топлива по сравнению с раздельной выработкой. Для одноконтурной ТПГУ (на базе GT13E2) наибольшее значение относительной экономии (по сравнению с

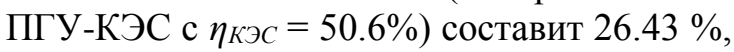
а для аналогичной двухконтурной ТПГУ $27.35 \%$.

4. ТПГУ более сложной структуры, для достижения максимального экономического эффекта, должны проектироваться на более высокие параметры пара. Использование одинаковых начальных параметров утилизационного контура ТПГУ, при различной структуре, позволяет унифицировать оборудование ТЭС, но приводит к снижению потенциальной экономии топлива до 0.4-0.5\%.

5. Изменение системных условий ведет к изменению относительной экономии топлива по сравнению с раздельной выработкой. При снижении эффективности замещающей КЭС с 50 до 40\% (при заданной эффективности замещающей водогрейной котельной), относительная экономия топлива по сравнению с раздельной выработкой двухконтурной ТПГУ с начальным давления пара контура ВД 9.8-10 МПа увеличится с 27.35 до $38.8 \%$.

\section{APPENDIX 1 (ПРИЛОЖЕНИЕ 1)}

${ }^{1}$ Fig. 1. Schematic diagram of a two-circuit cogeneration combined-cycle gas plant (1 gas turbine unit, 2 - heat recovery boiler, 3 deaerator, 4 - steam turbine, 5 - high-pressure feed pump, 6 - high-pressure drum, 7 - mains water heaters, 8 - water-water heat exchanger, 
9 - low-pressure feed pump, 10 - low-pressure drum).

${ }^{5}$ Fig. 2. Performance indicators of cogeneration two-circuit combined-cycle gas plant $(1-$ coefficient of utilization of heat of fuel, 2 - coefficient of specific electricity generation based on heat consumption).

${ }^{6}$ Fig. 3. The index of a relative fuel economy for single-circuit and two-circuit combinedcycle gas plant.

${ }^{2}$ Table 1. Performance of the GT13E2 gas turbine at an outdoor temperature of $-1.3^{\circ} \mathrm{C}$.

${ }^{3}$ Table 2. Summary results of calculation of the parameters of the mode of a two-circuit cogeneration combined-cycle gas plant.

${ }^{4}$ Table 3. Summary results of calculation of the efficiency indicators of a two-circuit cogeneration combined-cycle gas plant.

\section{Литература (References)}

[1] Chebotareva G.S. Metodicheskie osnovy otsenki konkurentosposobnosti energiticheskikh kompaniy [Methods for the Evaluation of the Competitiveness of Energy Companies]. Ekonomika regiona - Economy of Region, 2018, vol. 14, no. 1, pp. 190-201. doi:10.17059/2018-1-15.

[2] Makarov A.A., Veselov F.V., Makarova A.S., Novikova T.V., Pankrushina T.G. Strategic prospects of electric power industry in Russia. Thermal Power Engineering, 2017, no. 11, pp. 40-52. doi:10.1134/S0040601517110064.

[3] Makarov A.A., Mitrova T.A., Veselov F.V., Galkina A.A., Kulagin V.A. Perspectives of the electric power industry amid the transforming global power generation markets. Thermal Power Engineering, 2017, vol. 64, no. 10, pp. 703-714: doi:10.1134/S0040601517100081.

[4] Makarov A.A., Veselov F.V., Makarova A.S., Urvantseva L.V. Comprehensive assessment of Russia's electric power industry's technological transformation. Thermal Engineering, 2019, vol. 66, no. 10, pp. 687-701. doi:10.1134/S0040601519100045.

[5] Veselov F.V., Erokhina I.V., Makarova A.S., Khorshev A.A. Comprehensive assessment of the effective scope of modernization of thermal power plants to substantiate the rational structure of the generating capacities for the future until 2035. Thermal Engineering, 2017, vol. 64, no. $3, \quad$ pp. 161-169. doi:10.1134/S0040601517030107.

[6] Kalimullin L.V. Prioritetnye napravleniya razvitiya energeticheskikh kompaniy
[Priority development of energy-generating companies]. Vestnik Saratovskogo gosudarstvennogo socialnoekonomicheskogo yniversiteta - Bulletin of the Saratov State Socio-Economic University, 2018, vol. 2, no. 71, pp. 87-95.

[7] Filippov S.P., Dil'man M.D. CHP plants in Russia: the necessity for technological renovation. Thermal Engineering, 2018, vol. 65, no. 11, pp. 775-790. doi:10.1134/S0040601518110022.

[8] Kondrat'eva O.E., Roslyakov P.V., Loktionov O.A., Skobelev D.O., Guseva T.V., Åke M. Developing the costestimation technique when switching to best available power technologies. Thermal Engineering, 2019, vol. 66, no. 7, pp. 513520. doi:10.1134/S004060151907005X.

[9] Zamasz K., Kapłan R., Kaszyński P., Saługa P.W. An analysis of support mechanisms for new CHPs: the case of Poland. Energies, 2020, vol. 13, no. 21, p. 5635. doi:10.3390/en13215635.

[10] Ulanov V.L., Ulanova E.Y. Impact of external factors on national energy security. Journal of Mining Institute, 2019, vol. 238, no. $4, \quad$ pp. 474-480. doi:10.31897/pmi.2019.4.474.

[11] Kamchatova E., Salmina A. Analiz investitsionnikh programm energiticheskikh kompaniy [Analysis of the investment programs of energy companies]. Vestnik Universiteta - Bulletin of the University, 2018, no. 5, pp. 131-139. doi:10.26425/1816-4277-2018-5-131-139.

[12] Gosudarstvennyi doklad o sostoyanii energosberezheniya I povyshenii energeticheskoi effektivnosti $v$ Rossiiskoi Federatsii [State Report on the state of Energy Saving and Energy efficiency improvement in the Russian Federation]. Available https://minstroyrf.gov.ru/docs/18226. (accessed 25.02.2021).

[13] Olkhovskii G.G. Combined cycle plants: yesterday, today and tomorrow (review). Thermal Engineering, 2016, vol. 63, no. 7, pp. 488-494. doi:10.1134/S0040601516070041.

[14] Lenev S.N., Radin Yu.A., Golov P.V., Konovalov V.I., Istomov A.I. Modernizatsiya bloka PGU-420 TETS PAO «Mosenergo» s povysheniem effektivnosti raboty bloka [Modernization and increase of an overall performance efficiency of CCGT420 at CHP-20 of the PJSC «Mosenergo»]. Elektricheskie stantsii - Electric power stations, 2020, vol. 5, no. 1066, pp. 20-24.

[15] Teplov B.D., Burov V.D. The extension of the operational range of combined-cycle 
power plant with a triple-pressure heat recovery steam generator. Journal of Physics: Conference Series, 2017, p. 012208. doi:10.1088/17426596/891/1/012208.

[16] Teplov B.D., Radin Yu.A. Improving flexibility and economic efficiency of CCGT units' operation in the conditions of the wholesale electricity market. Thermal Engineering, 2019, vol. 66, no. 5, pp. 323330. doi:10.1088/1742-6596/1260/5/052005.

[17] Larin B.M. Water-chemistry and its utility systems in CCP power units (Review). Thermal Engineering, 2018, vol. 65, no. 1, pp. $39-44$. doi:10.1134/S0040601517120059.

[18] Polonsky V.S., Tarasov D.A., Gorr D.A. Configuration of evaporators for oncethrough heat recovery steam generators of combined-cycle units. Thermal Engineering, 2019, vol. 66, no. 5, pp. 340-349.

[19] Sultanguzin I.A., Fedyukhin A.V., Yavorovsky Y.V., Voloshenko E.V., Kurzanov S.Y., Stepanova T.A., Tumanovsky V.A., Ippolitov V.A., Zakharenkov E.A. An analysis of the prospects for coal-fired thermal power station reconstruction on the basis of coal gasification and a combined-cycle unit. Thermal Engineering, 2020, vol. 67, no. 7, pp. 451-460.

[20] Filimonova A.A., Chichirov A.A., Chichirova N.D., Batalova A.A., Arakelyan E.K. Physicochemical Analysis of Organic Impurities in the Coolant of Combined Heat and Power Plants Equipped with CombinedCycle Power Installations. Thermal Engineering, 2020, vol. 67, no. 5, pp. 314319. doi:10.1134/S0040601520050067.

[21] Borisov Y., Fominykh N., Ramazanov E., Popel O. Analysis of the compressorless combined cycle gas turbine unit performance efficiency in district heating systems. E3S Web of Conferences, 2020, vol. 209, no. 03008, p.8. doi:10.1051/e3sconf/202020903008.

[22] Toscano C.F., Martin-del-Campo C., Moeller-Chavez G., Leon de los Santos G., François J.-L., Fernandez D.R. Life cycle assessment of a combined-cycle gas turbine with a focus on the chemicals used in water conditioning. Sustainability, 2019, vol. 11, no. 10, pp. 2912. doi:10.3390/su11102912.

[23] Galashov N.N., Tsibulskiy S.A. Parametricheskii analiz skhemy parogazovoi ustanovki s kombinatsiei trekh tsiklov dlya povisheniya KPD pri rabote $\mathrm{v}$ severnykh gazodobyvayushikh raionakh [Parametric analysis of the diagram of the combined cycle gas turbine with a combination of three cycles for improving efficiency when operating in northern gas producing areas]. Izvestiya Tomskogo Politekhnicheskogo Universiteta Inziniring Georesursov Bulletin of the Tomsk Polytechnic University. Geo Assets Engineering, 2019, vol. 330, no. 5, pp. 44-55. doi:10.18799/24131830/2019/5/274.

[24] Almutairi A., Pilidis P., Al-Mutawa N. Energetic and exergetic analysis of combined cycle power plant: Part-1 Operation and performance. Energies, 2015, vol. 8, no. 12, pp. 14118-14135. doi:10.3390/en81212418.

[25] Keshavarzian S., Gardumi F., Rocco M.V., Colombo E. Off-Design modeling of natural gas combined cycle power plants: an order reduction by means of thermoeconomic input-output analysis. Entropy, 2016, vol. 18, no. 71. doi:10.3390/e18030071.

[26] Ol'khovskii G.G., Trushechkin V.P., Ageev A.V., Tuz N.E., Teplov B.D., Rozhkov A.D. Fuel utilization monitoring techniques for large-capacity combined cycle power plants. Thermal Engineering, 2019, vol. 66, no. 5, pp.

316-322. doi:10.1134/S0040601519050070.

[27] Kler A.M., Marinchenko A.Yu., Potanina Yu.M. Optimizatsionnye issledovaniya parogazovoi ustanovki s gazifikatsiei uglya I vysokotemperaturnym podogrevom dut'evogo vozdukha [Optimization studies of a combined-cycle gas plant with coal gasification and high-temperature blast air heating]. Izvestiya Tomskogo Politekhnicheskogo Universiteta Inziniring Georesursov - Bulletin of the Tomsk Polytechnic University. Geo Assets Engineering, 2019, vol. 330, no. 3, pp. 7-17. doi:10.18799/24131830/2019/3/159.

[28] Burov V.D., Soiko G.V., Dudolin A.A., Makarevich E.B., Zakharenkov E.A., Kovalev D.A. Metodika optimizatsii struktury i parametrov moshchnikh parogazovykh TES [Methodology of optimization of structure and parameters of powerful steam-gas thermal power plants]. Energosberezhenie $i$ vodopodgotovka Energy saving and water treatment, 2013, vol. 5, no. 85 , pp. 37-42.

[29] Moshkarin A.V., Mel'nikov Yu.V., Zhamlikhanov T.A.., Shomov E.V. Optimizatsiya davlenii u utilizatsionnoi PGU dvuh davlenii $\mathrm{s}$ uchetom tekhnicheskih ogranichenii [Pressure optimization in threecircuit disposal CCP subject to engineering limitation]. Vestnik Ivanovskogo Gosudarstvennogo Energeticheskogo Universiteta - Bulletin of the Ivanovo State Power Engineering University, 2007, no. 4. 
[30] Kalyutik A.A., Treschev D.A., Trescheva M.A. Ispol'zovanie pokazatilya otnositel'noi economii topliva dlya optimizatsii parametrov teploficatsionnoi PGU [Using the parameter of relative fuel savings to optimize the parameters of cogeneration CCGT]. Materialovedenie. Energetika Materials Science. Power Engineering, 2020, vol. 26, no. 4, pp. 51-63. doi:10.18721/JEST.26404.

[31] Balynin I.V. Otsenka rezul'tativnosti investitsionnih proektov: pravila. pokazateli $i$ poryadok ih rascheta [Assessing the effectiveness of investment projects: rules. indicators and their calculation procedure]. Ekonomicheskii analiz: teoriya i praktika Economic analysis: theory and practice, 2016, vol. 6, no. 453, pp. 26-41.

[32] Nikolenko T.Yu., Tarasova E.V. Sistema sbalansirovannykh pokazatelei instrumentarii otsenki effektivnosti innovatsionnykh proektov [The system of balanced indicators evaluation and the tools for evaluating the effectiveness of innovative projects]. Nauchno-tekhnicheskie vedomosti SPbGPU. Ekonomicheskie nauki SPbPU Journal. Economics, 2016, vol. 6, no. 256, pp. 228-235. doi:10.5862/JE.256.21.

[33] SP 131.13330.2018. Stroitel'naya klimatologiya [Body of rules 131.13330.2018. Building climatology]. Available https://minstroyrf.gov.ru/docs/18226. (accessed 25.02.2021).

[34] Tsanev S.V., Burov V.D., Remezov A.N. Gazoturbinnye i parogazovye ustanovki teplovykh elektrostantsii [Gas-turbine and combined-cycle installations of thermal power plants]. Moscow, 2009. 584 p.

\section{Сведения об авторах.}
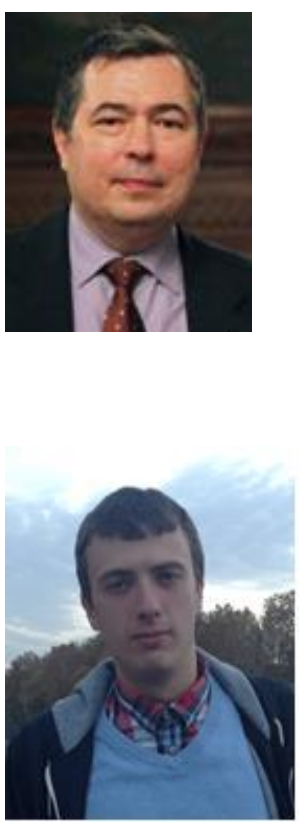

Калютик Антонович

к.т.н., Доцент,

Петра Великого.

Научные

интересы:

атомная и тепловая энергетика, парогазовые установки, теплофикация E-mail: kalyutik@yandex.ru

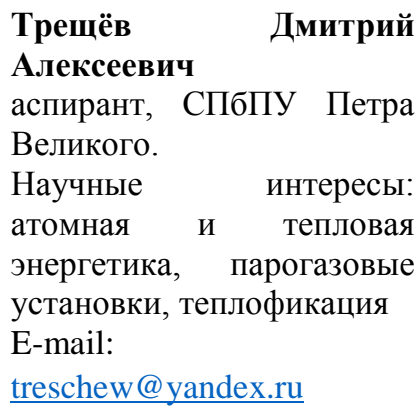

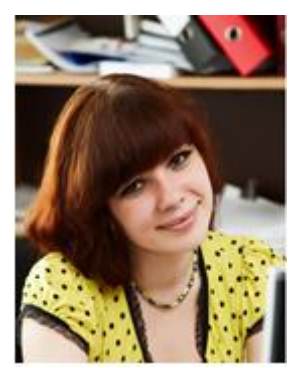

Трещёва

Алексеевна

к.т.н., СПбПУ Петра

Великого.

Научные интересы: атомная и тепловая энергетика, парогазовые установки, теплофикация E-mail:

milana.treshcheva@gmail.com 